ICATPP Como 2013

\title{
A Scintillating Fibre Tracker for the LHCb Upgrade
}

\author{
Robert Ekelhof, TU Dortmund \\ on behalf of the \\ LHCb Collaboration
}




\section{Contents}

- LHCb \& SciFi Upgrade

- Fibre R\&D

- SiPM R\&D

- Modules

- Electronics

- Simulation 


\section{Related Talks of Interest}

- Wednesday plenary

- "The LHCb upgrade "

- Eric Van Herwijnen (CERN)

- "Recent advances in the development and applications of scintillating fibres in HEP and astroparticle physics experiments"

- Frédéric Blanc (EPFL, Switzerland)

- Monday, Tracker and Applications I

- $\quad$ "The Performance and Radiation Hardness of the Outer Tracker Detector for LHCb"

- Evelina Gersabeck (Universität Heidelberg)

- "Tracking and Alignment of the LHCb detector"

- Maurizio Martinelli (Nikhef, Amsterdam) 


\section{LHCb \& SciFi Upgrade}




\section{$\mathrm{LHCb}$}

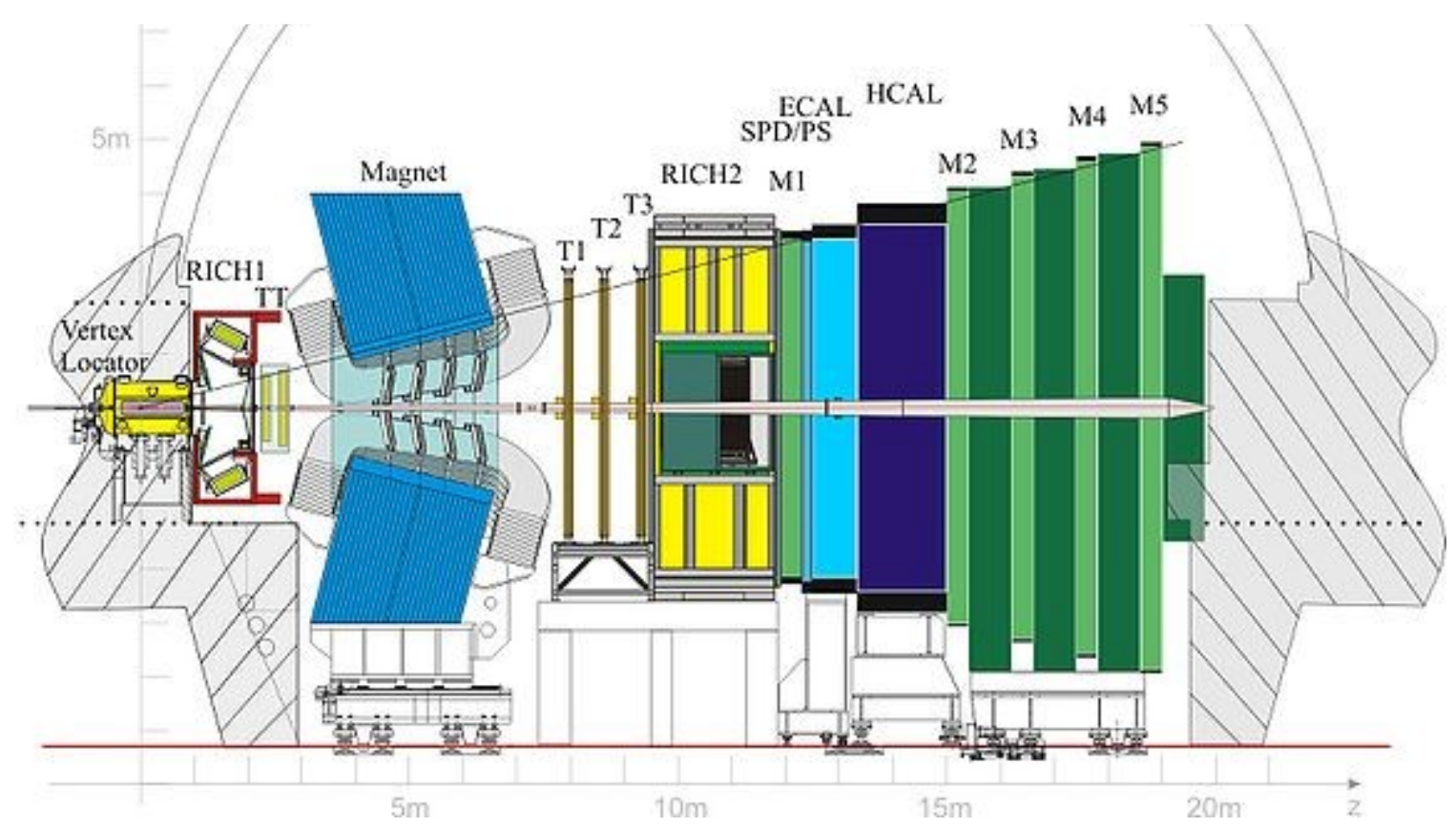

- single-arm spectrometer (10-300mrad)

- dedicated to heavy flavour physics at the LHC, looks for

- indirect evidence of new physics in CP violation

- rare decays of beauty and charm hadrons 


\section{LHCb Upgrade}

- current detector optimised for lower luminosity than LHC could deliver

- one interaction per bunch crossing

- instant luminosity $2 \mathrm{E} 32 \mathrm{~cm}^{-2} \mathrm{~s}^{-1}$

(design specification)

- upgrade of whole detector is planned for LS2 in 2018/19

- increase instant luminosity up to $2 \mathrm{E} 33$ to record a total integrated luminosity of $50 \mathrm{fb}^{-1}$

- great change in Trigger, change to full software trigger

- this requires $40 \mathrm{MHz}$ readout of FE electronics

- several subdetectors will be replaced 


\section{SciFi@ LHCb}

- upgrade of downstream tracking stations T1-T3, since the occupancy in the current Outer Tracker would be too high

- two alternatives:

- enlarged silicon IT + drifttube OT

- SciFi CT (or full area FT without OT)

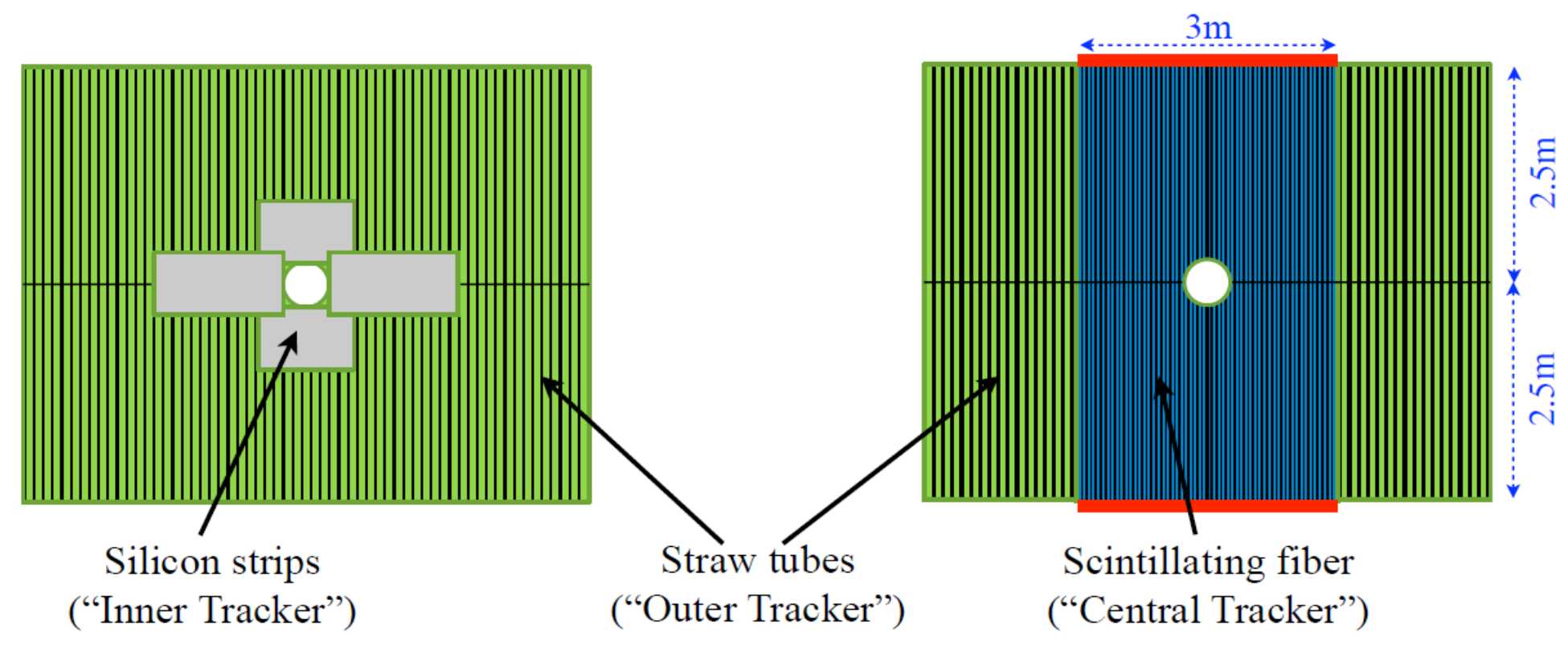




\section{SciFi@ LHCb}

- $250 \mu \mathrm{m}$ diameter scintillating fibres (pitch $280 \mu \mathrm{m}$ )

- read out by multi-channel Silicon-Photomultipliers (SiPM) (pitch 250 $\mu$ m)

- 5 layers for sufficient light output

- $5 \mathrm{~m}$ acceptance is split in two halfs of $2.5 \mathrm{~m}$ fibres

- SiPM readout on the outer edge

- mirrored ends at the inner edge
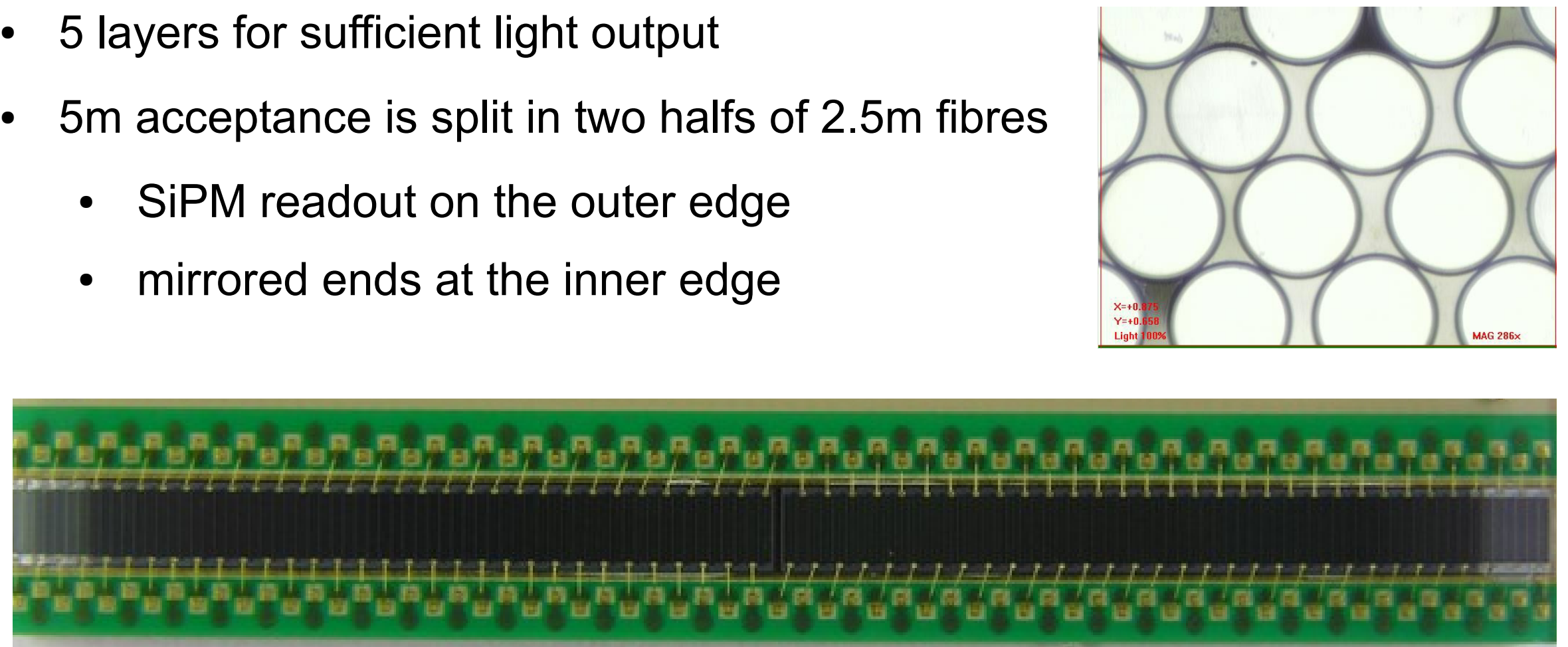


\section{SciFi @ LHCb}

- how does it work?

- hits in neighbouring channels form cluster

- hit position calculated by weighted sum

- different thresholds can be set for best SNR

- requirements

- high hit detection efficiency (98-99\%)

- spatial hit resolution of

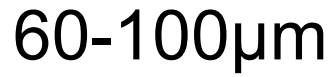

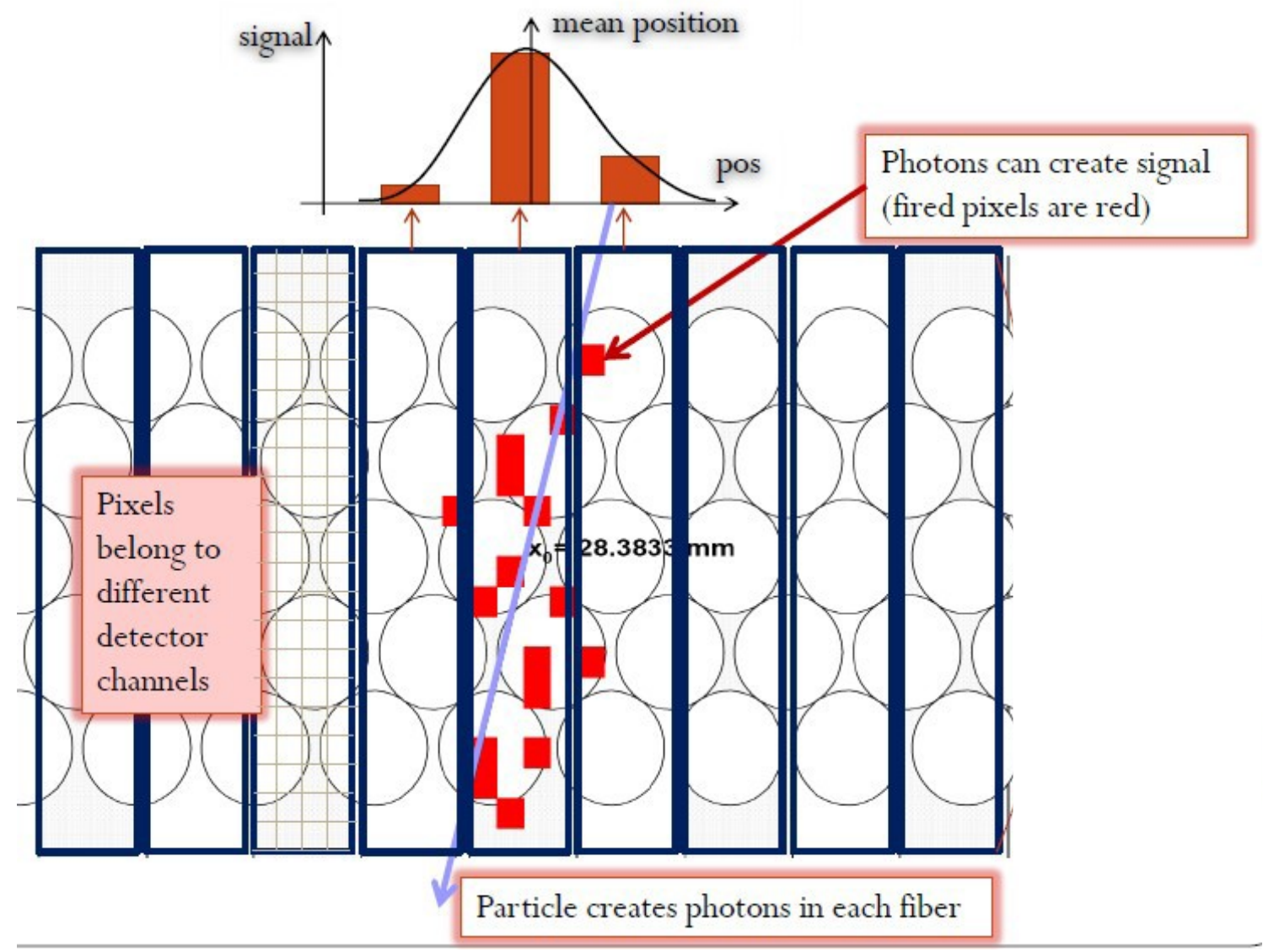

- low material budget 


\section{Radiation Environment}

- detector components have to withstand the harsh LHC environment

- SiPMs

- $1 \mathrm{MeV}$ neutron equivalent of $6 \mathrm{E} 11 / \mathrm{cm}^{2}$

- dose <100Gy

- may be lowered with shielding

- fibres

- dose up to $30 \mathrm{kGy}$ near beam pipe

- falls rapidly to outer edges
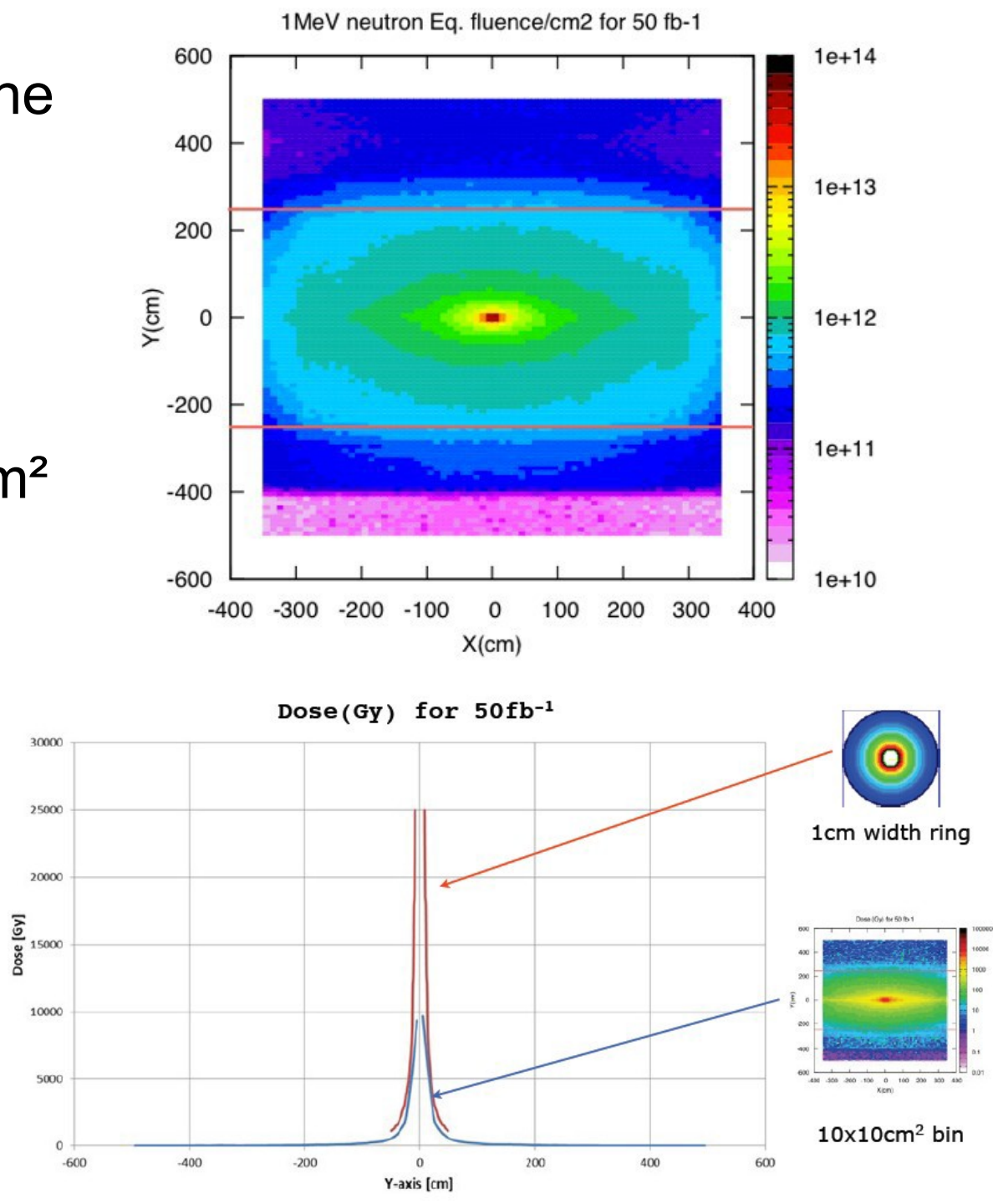
Fibre R\&D 


\section{Scintillating Fibres}

- baseline fibre Kuraray SCSF-78MJ

- scintillating core \& two claddings

- "scintillation" known to be radiation hard

- attenuation length effected by irradiation

- 3HF (green) fibres more radiation hard, but lower light yield and longer decay time

- existing literature inconsistent concerning quantative results

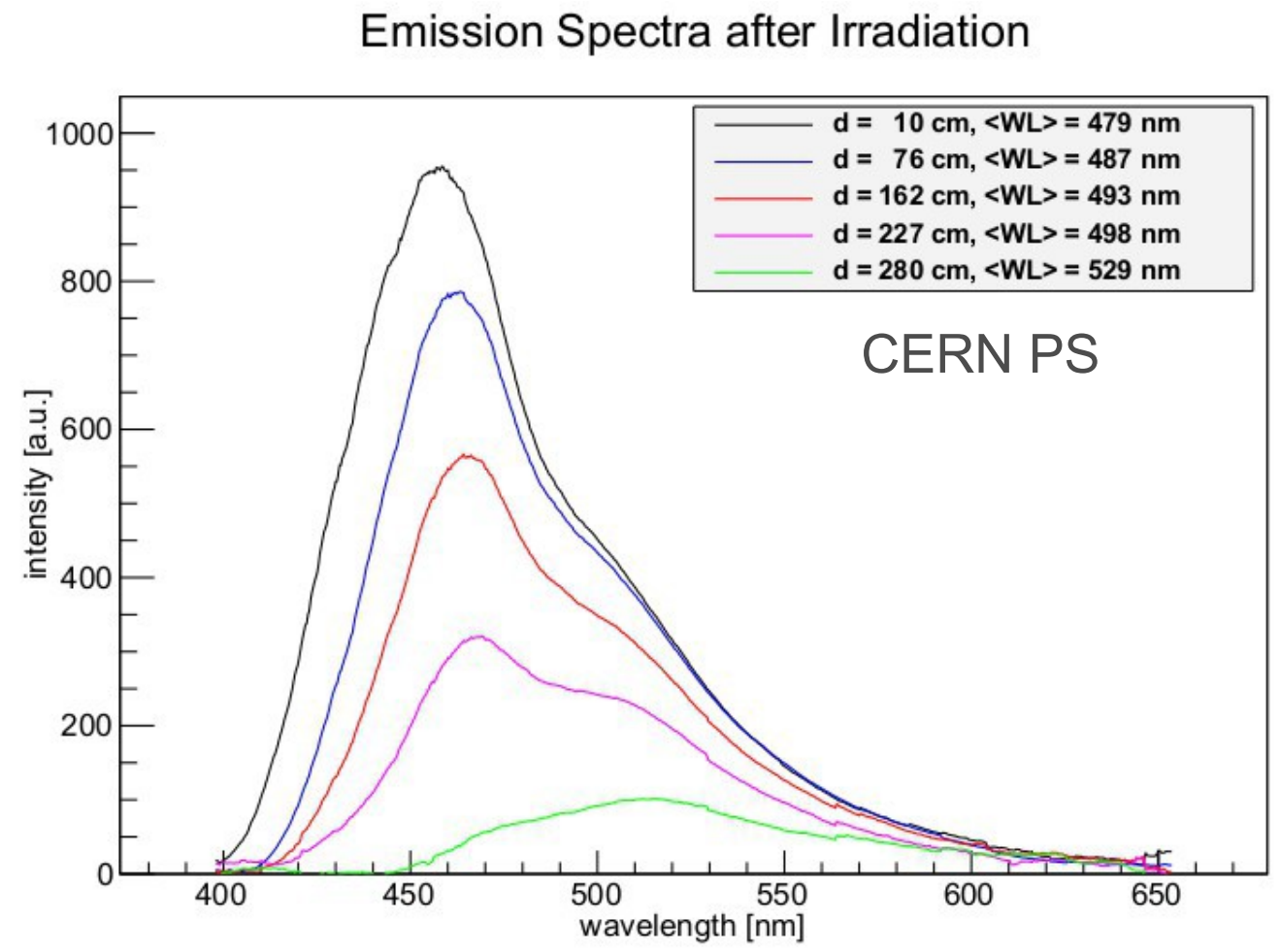




\section{Irradiation Campaigns}

- different irradiations where performed

- in situ LHCb ( $\approx 0.1 \mathrm{kGy})$

- CERN PS (3kGy, 22kGy)

- Karlsruhe (10kGy, 40kGy)

- Munich (100kGy)

- attenuation of light measured with excitation by UV-LED or radioactive

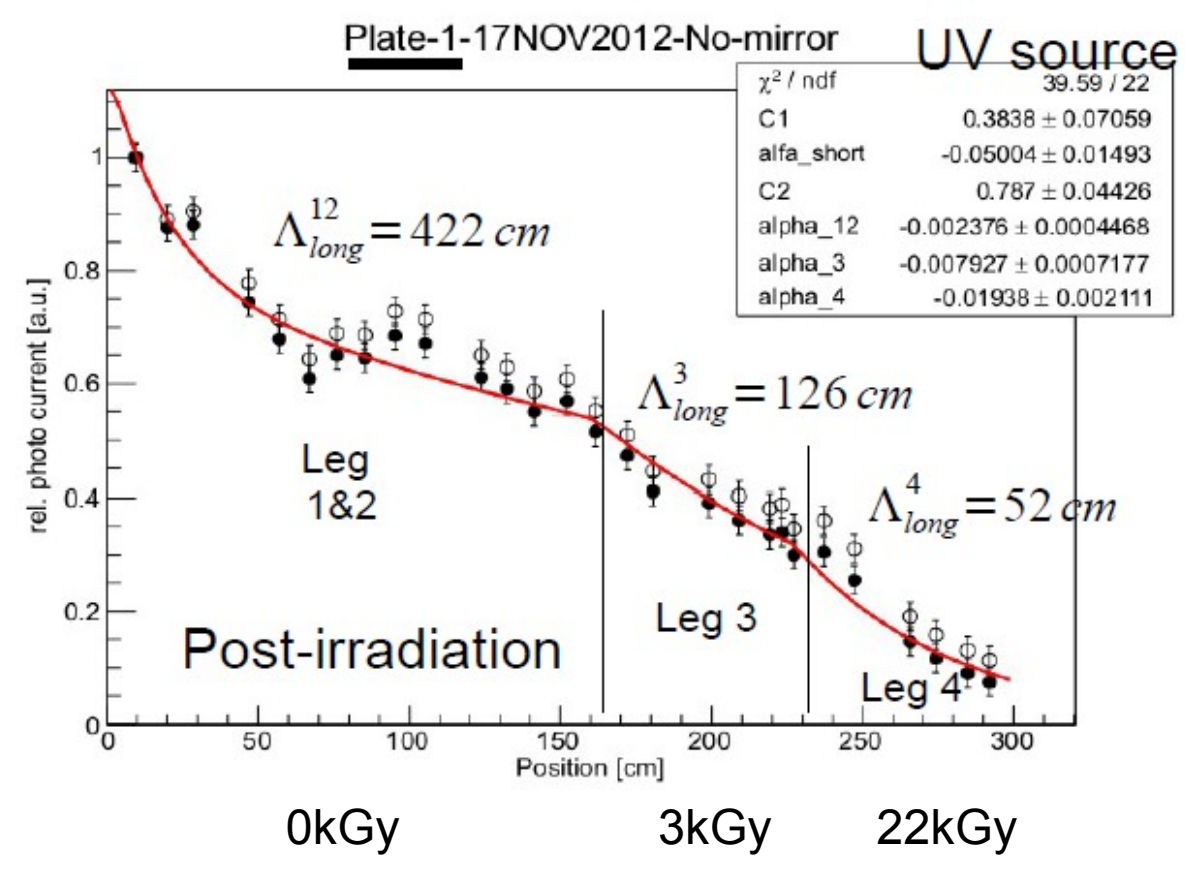
sources

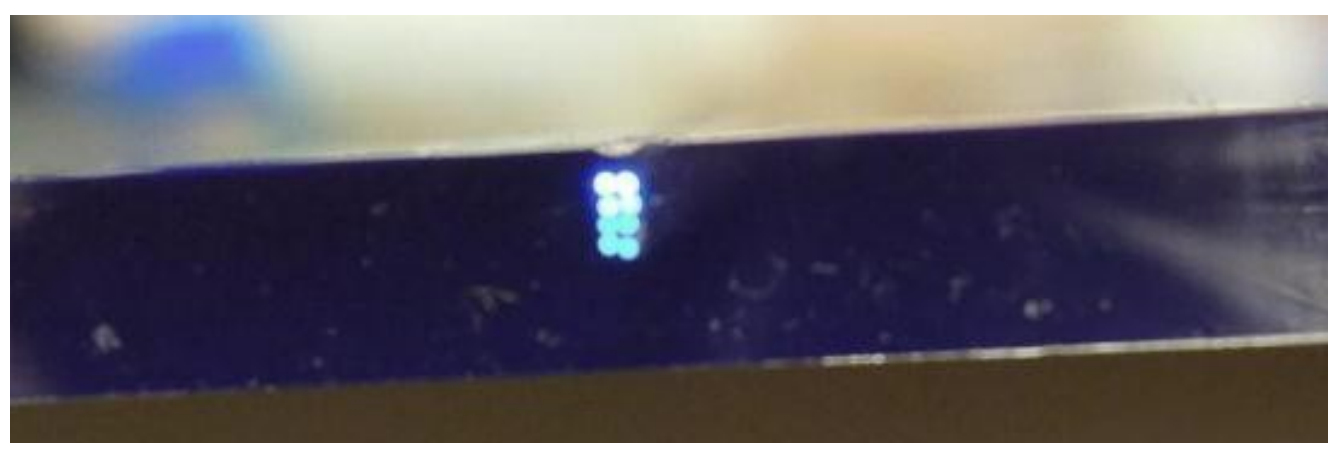




\section{Irradiation Results}

- the data of the different campaigns where analysed wavelength dependend

- the attenuation length as function of the dose is fitted with the empirical model developed by Hara et al. ${ }^{*}$

- although the damage occurs quickly (in dose), it mainly affects the center

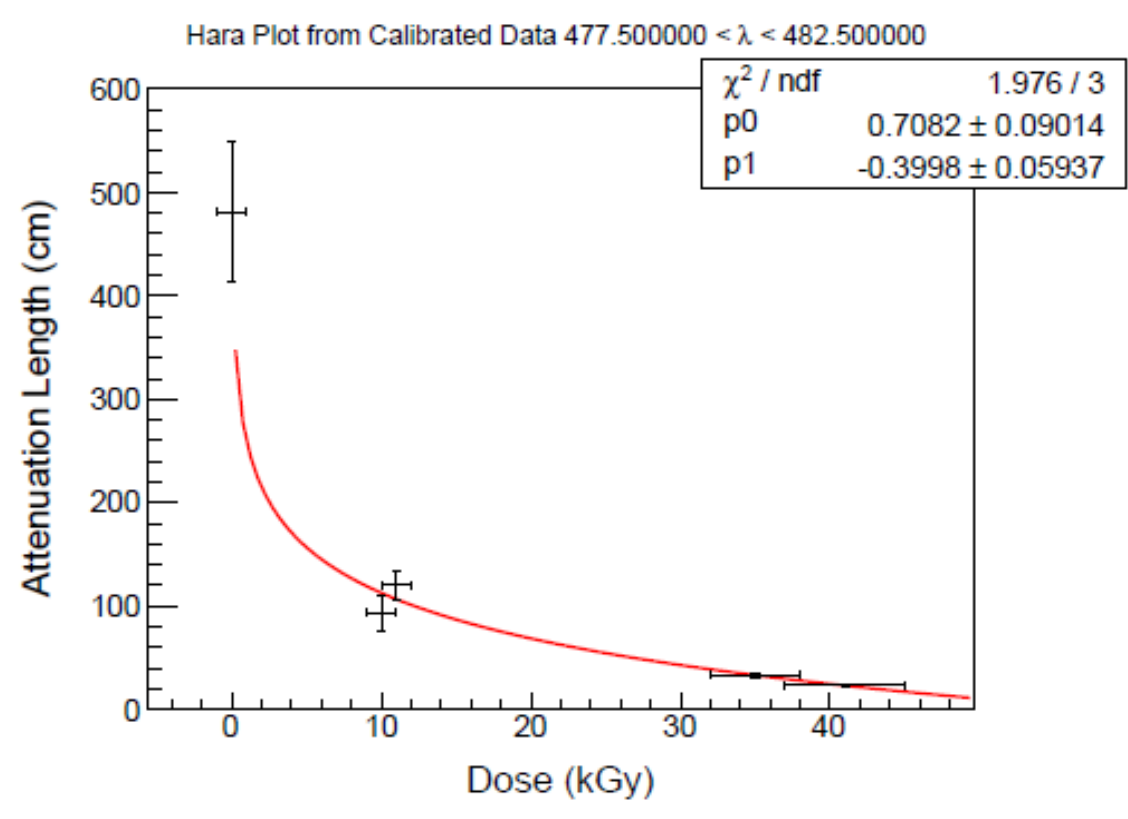

- new physically motivated models are tested to fit the data and are used for simulation of light transport

- $\quad$ K. Hara, K. Hata, S. Kim, M. Mishina, M. Sano, Y. Seiya, K. Takikawa, M. Tanaka, and K. Yasuoka. „Radiation hardness and mechanical durability of kuraray optical fibers." Nuclear Instruments and Methods in Physics Research Section A: Accelerators, Spectrometers, Detectors and Associated Equipment, 411(1):31 - 40, 1998.

Expected relative light yield: after 0 and $50 \mathrm{fb}-1$ (with/without mirror $\mathrm{R}=0.7$ ) (model of Hara et al.)

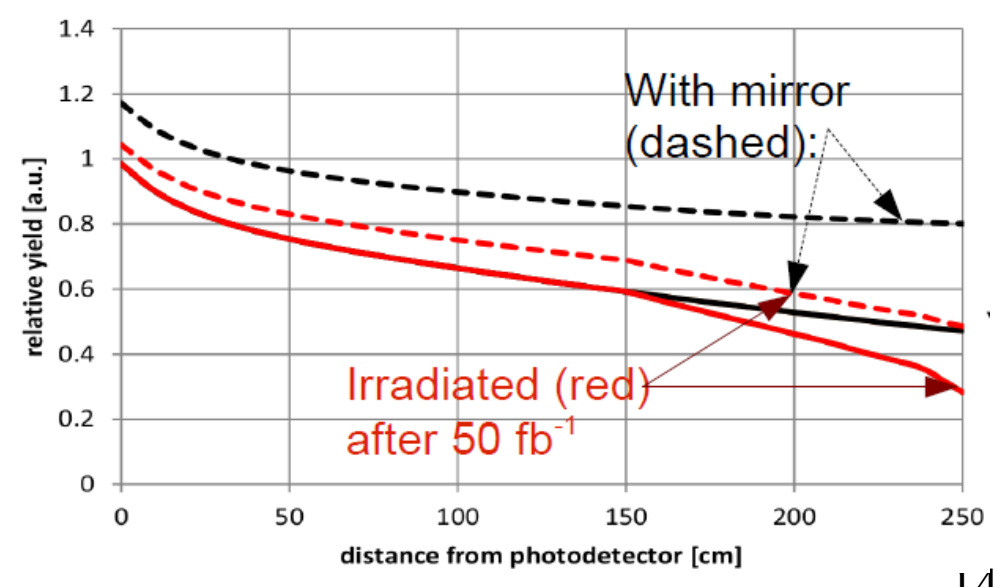




\section{SiPM R\&D}




\section{Characterisation}

- $\quad$ SiPM = array of Geiger mode avalanche photodiodes ("pixels")

- number of fired pixels gives the number of photons

- multichannel SiPMs by Hamamatsu and KETEK

- noise simulation to test the effect of different parameters

- primary noise

- temperature

- neutron dose

- crosstalk

- after-pulse

- shaping time

- thresholds

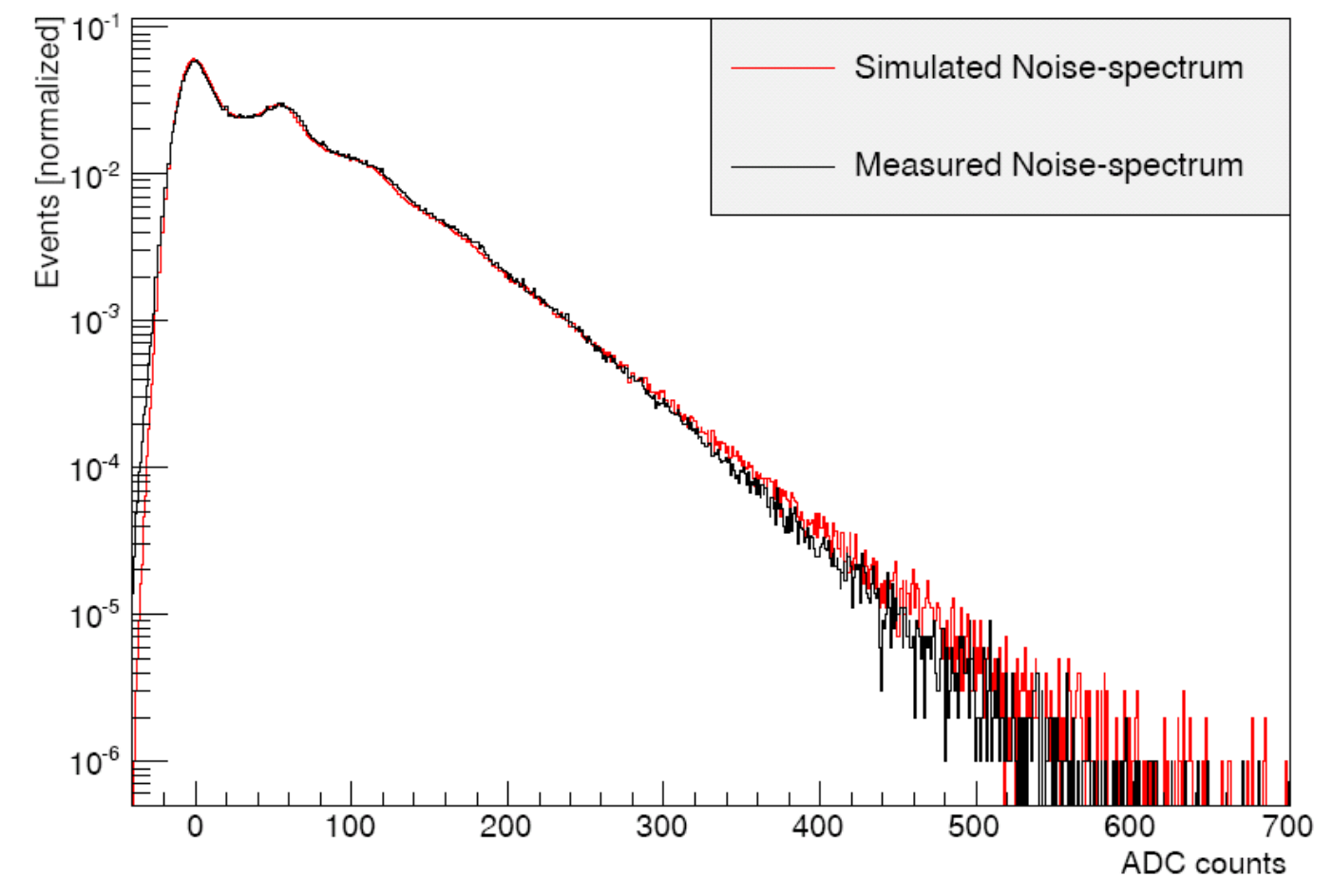




\section{Irradiation}

- several irradiation campaigns (accelerators, in situ, radioactive sources)

- noise increases linearly with dose

- noise reduced by factor 2 every $10^{\circ} \mathrm{C}$

- cool SiPMs to $-40^{\circ} \mathrm{C}$ (maybe $\left.-50^{\circ} \mathrm{C}\right)$

- heated during Technical Stop for annealing

- annealing at ambient $\mathrm{T}$ too slow for TS

- at $40^{\circ} \mathrm{C}->$ few days

- at $60^{\circ} \mathrm{C}->$ few hours

normalised dark currents

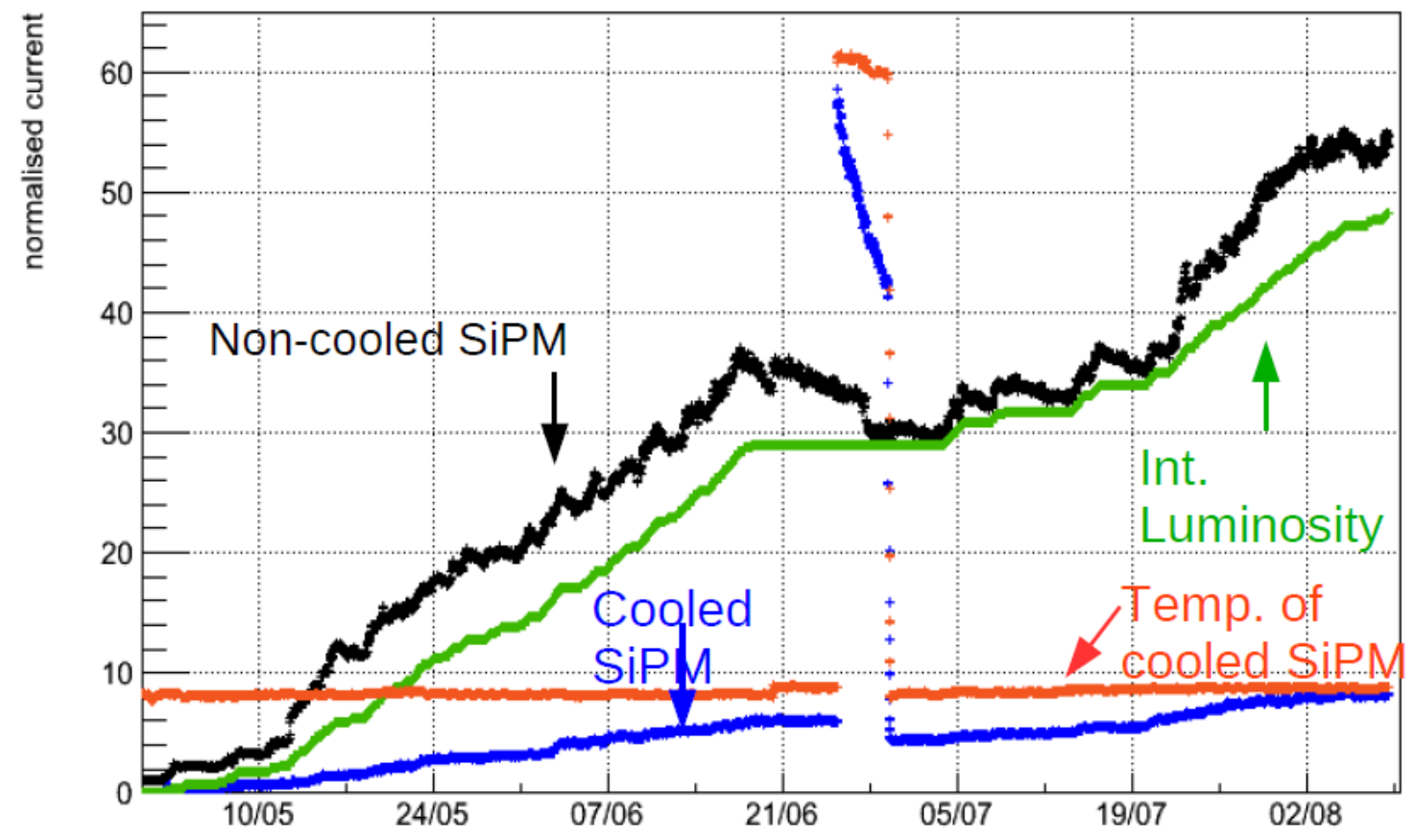




\section{Crosstalk \& Improvements}

- pixel-pixel x-talk

- fine for signal

- brings dark noise above 1 p.e.

- lowering crosstalk with trenches

- additional improvements:

- higher PDE

- green shifted sensitivity

- optimised pulse shape

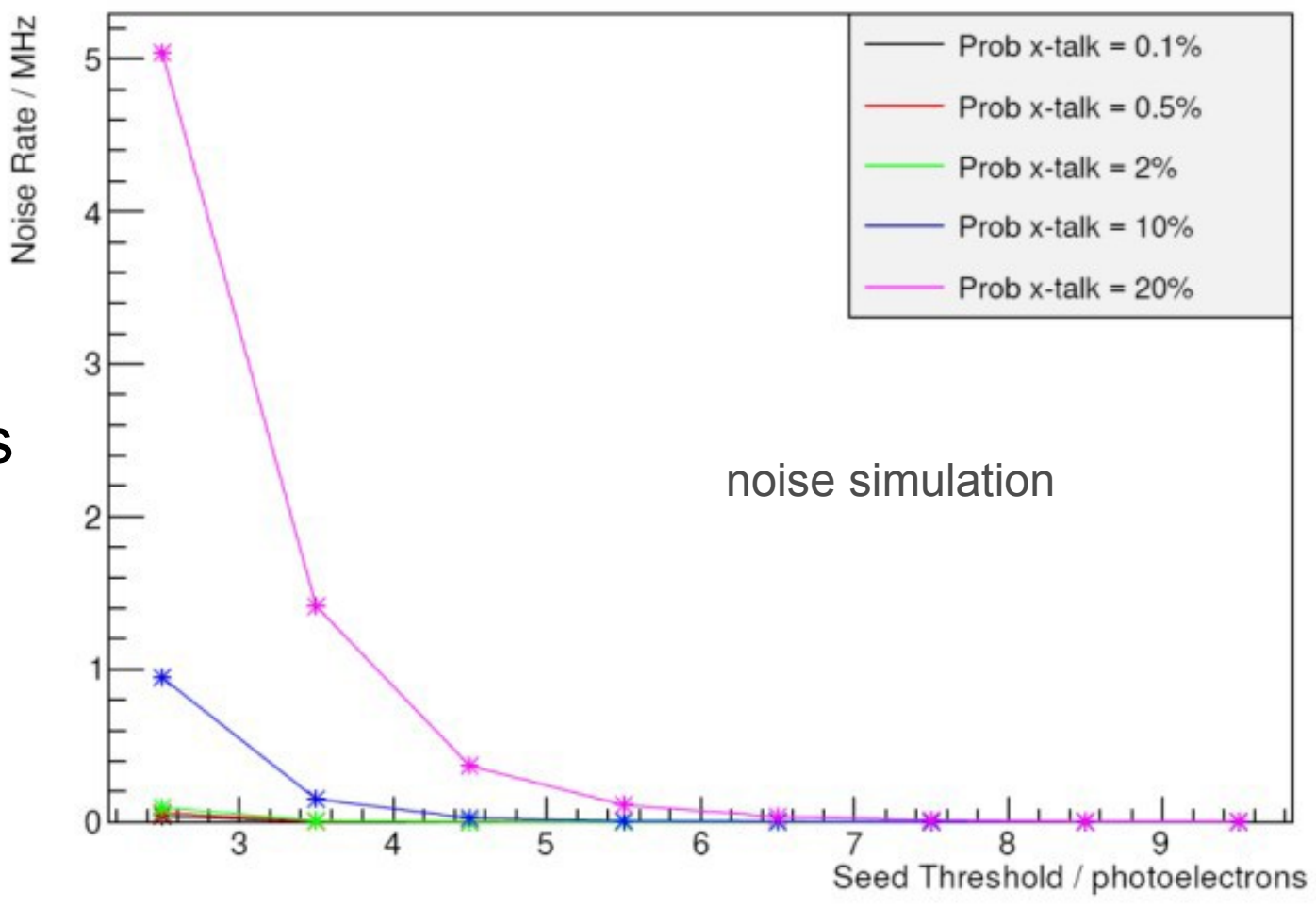




\section{Viability Assessment}

- Technical Board of LHCb asked for a viability assessment of the SciFi Tracker technology for $\mathrm{LHCb}$

- main goal was to judge the radiation hardness of the fibres and SiPMs

- it was concluded that it's a viable technology option for the LHCb upgrade tracker system (March2013)

next step is to build demonstrator modules to prove

- precise fibre layers

- building of large size modules

- coupling to SiPMs

- Cooling

- technology choice (silicon strips + drift tubes vs SciFi) in November 


\section{Modules}




\section{Overview}

- LHCb downstream tracking: 3 stations with 4 layers of $6 \times 5 \mathrm{~m}$

- 4 layers: $0^{\circ},+5^{\circ},-5^{\circ}, 0^{\circ}$ stereo angle

- one SciFi module:

- $53 \mathrm{~cm}$ wide, $5 \mathrm{~m}$ long (high)

- 2x4 fibre mats

- 2x16 SiPMs (with 128ch each)

- 2x one cooling bar

- $10 \mathrm{k} \mathrm{km}$ of scintillating fibre

- 600k channels

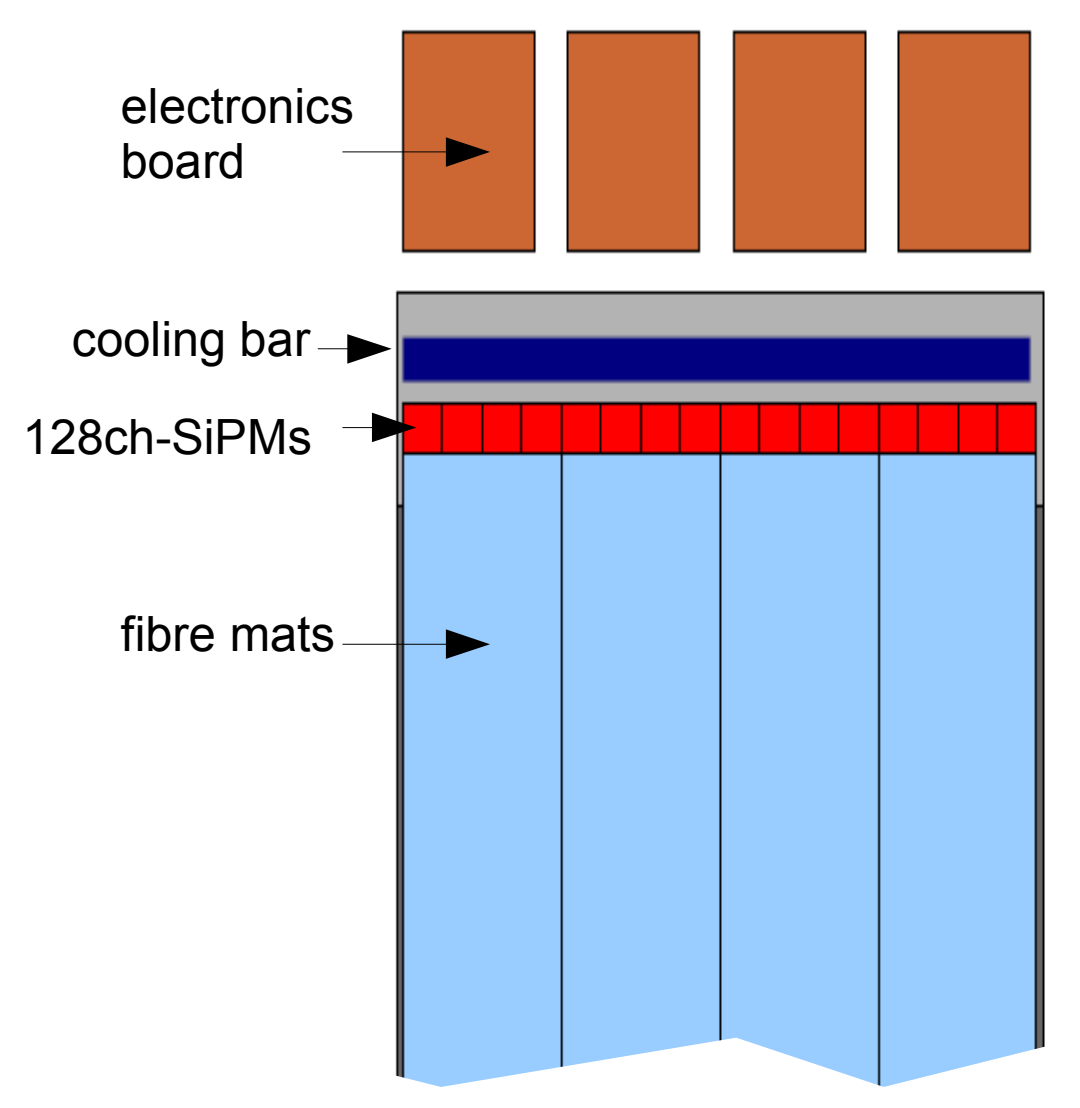




\section{Fibre Mats}

- production method for a fibre mat inspired by production of fibre modules by RWTH Aachen for the PEBS experiment

- fibres are wound on a wheel ( $1 \mathrm{~m}$ diameter) with a $280 \mu \mathrm{m}$ thread

- glue is placed on the fibre right before it touches the wheel

- the $1^{\text {st }}$ layer of fibres is used as thread for the $2^{\text {nd }}$ layer and so forth

- after glue has cured "enough", the mat is cut and taken off to be flattened

- the sides along the fibres are cut to achieve a good edge

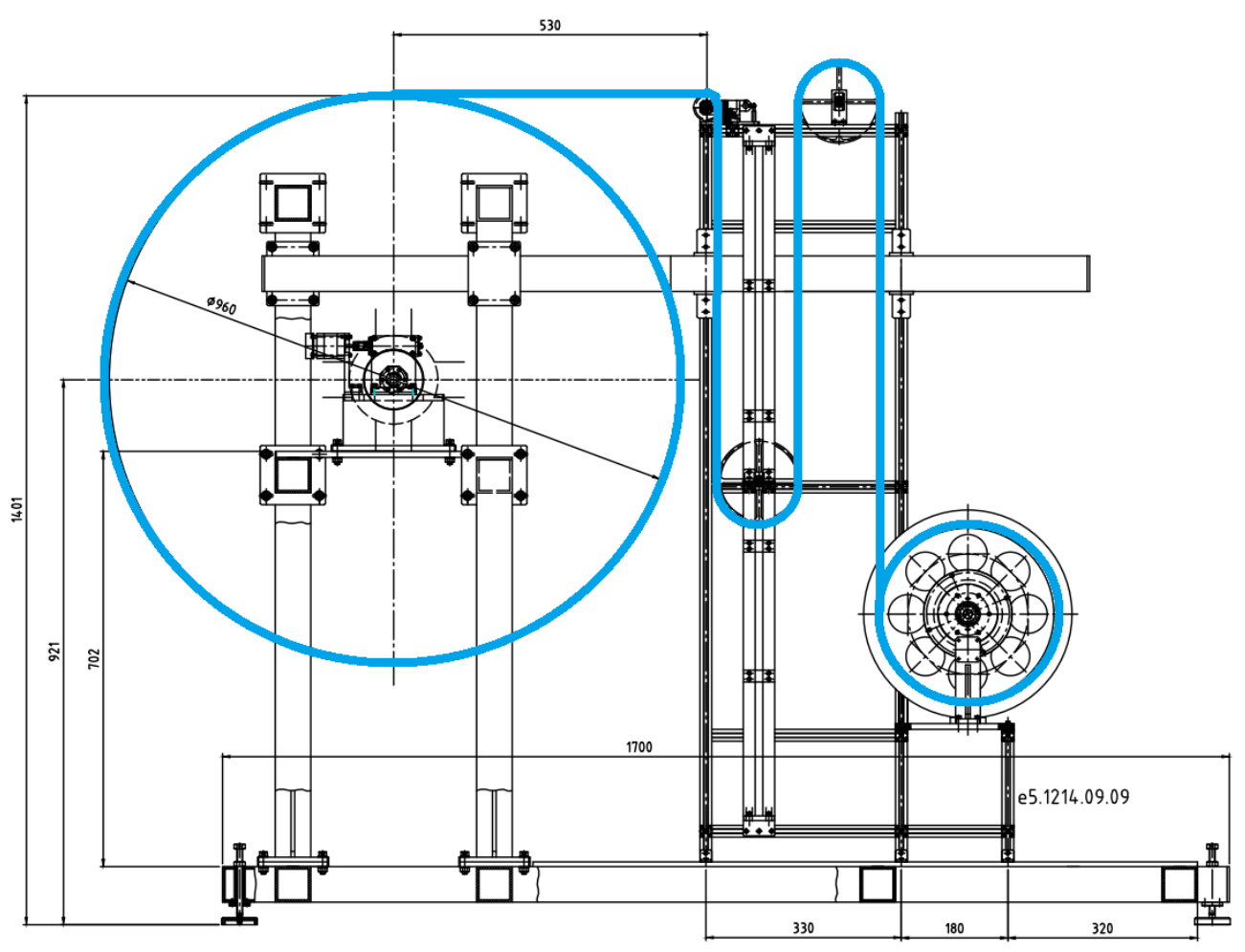




\section{fibre mats}

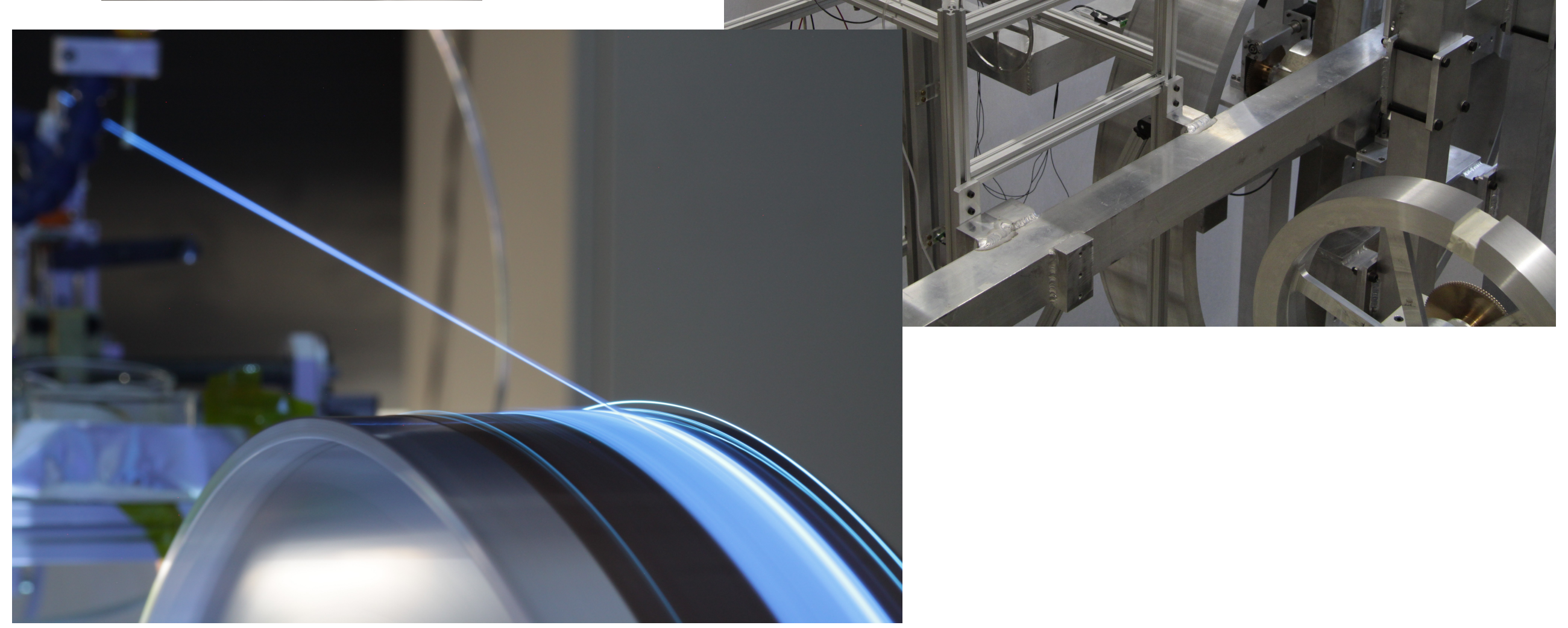




\section{Fibre Mats}

- alternative to threads on the wheel is investigated

- Kapton substrate with groove structure which is positioned on the wheel

- photoimageable coverlay (epoxy-like, DuPont)

- the Kapton stays attached to the fibre mat and can provide alignment marks
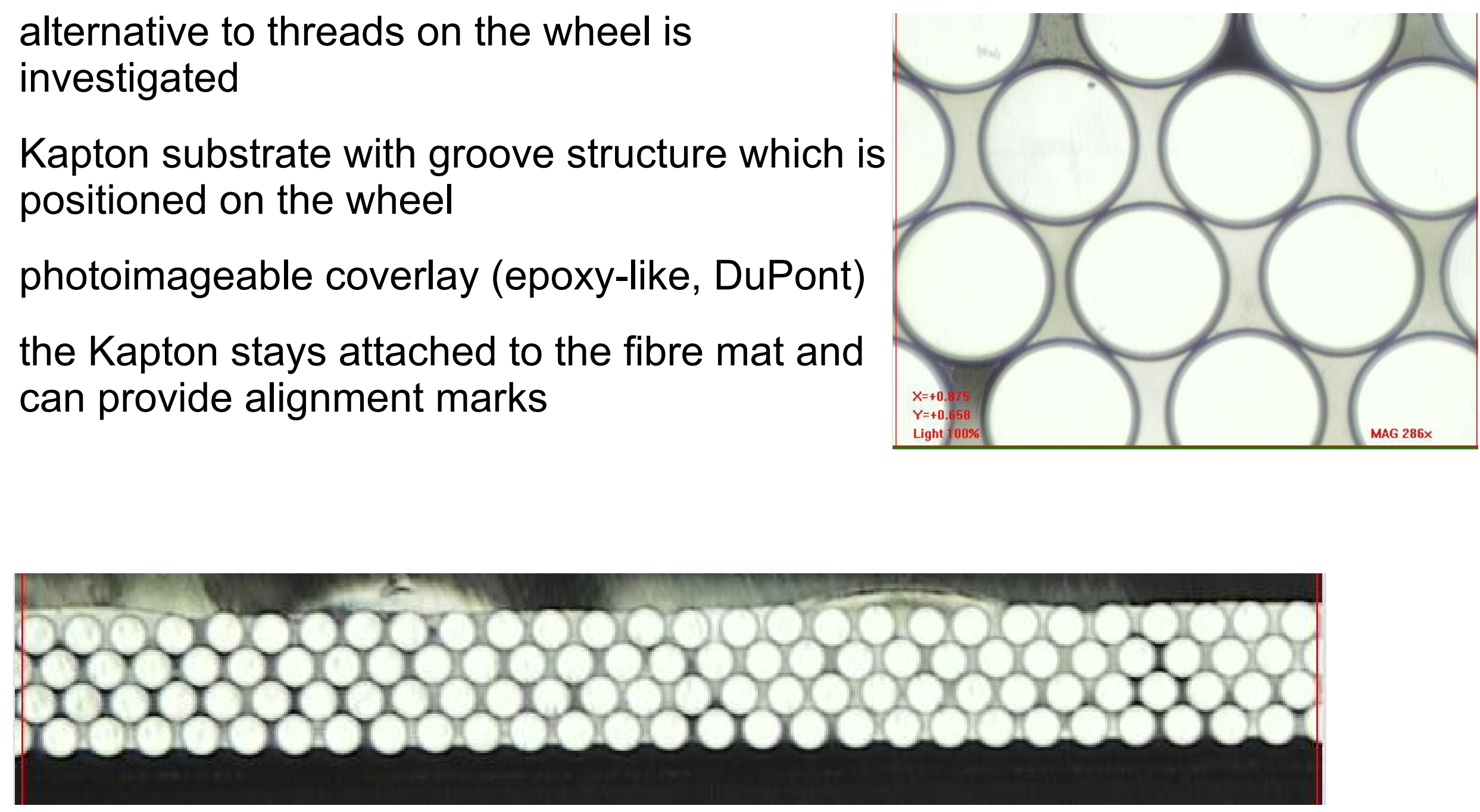


\section{Support Structure}

- fibre mats are positioned in the middle of a sandwich structure

- support panel is not devided, $5 \mathrm{~m}$ long

- honeycomb with carbon fibre skins (+light tight foil)

- radiation length $\approx 0.6 \%$ ( $+\approx 0.3 \%$ for fibres $)$

- SiPMs mounted to "end piece"

- alignment pins on the fibre mats ensure the straightness

carbon fibre

1 honeycomb

fibre mat 


\section{Support Structure}

- "End Cap"

- top/bottom end of module with connection to FE board

- enclosure for cold volume

- connection from fibres to SiPMs to cooling pipe

- mounting point of module

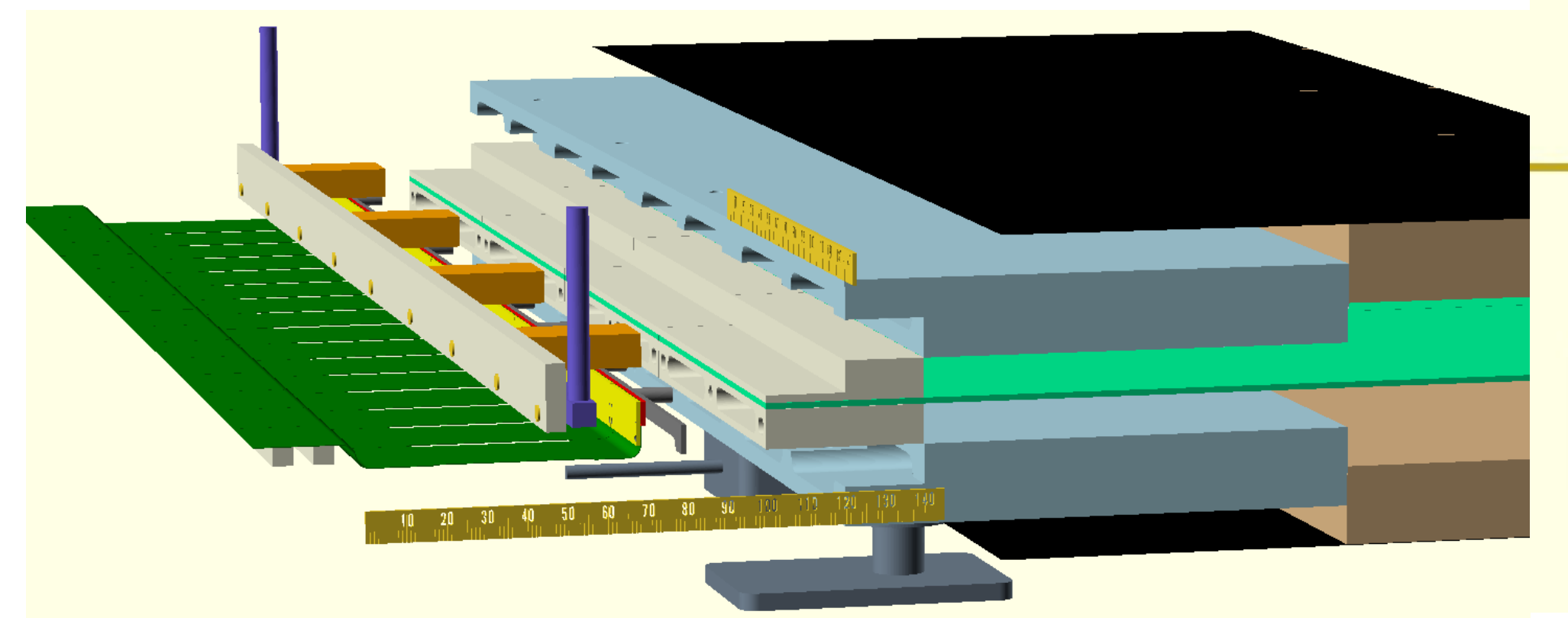




\section{SiPM Cooling}

- cool down to $-40^{\circ} \mathrm{C}$

- cooling power dominated by heat leaks

- different technologies under investigation

- liquid cooling (2-phase, single-phase)

- air cooling

- peltier cooling

- simulations performed to estimate heat load and evaluate the influence of different module geometries

- tests with mockups performed and ongoing

- in parallel: tests of thermally cycled fibres

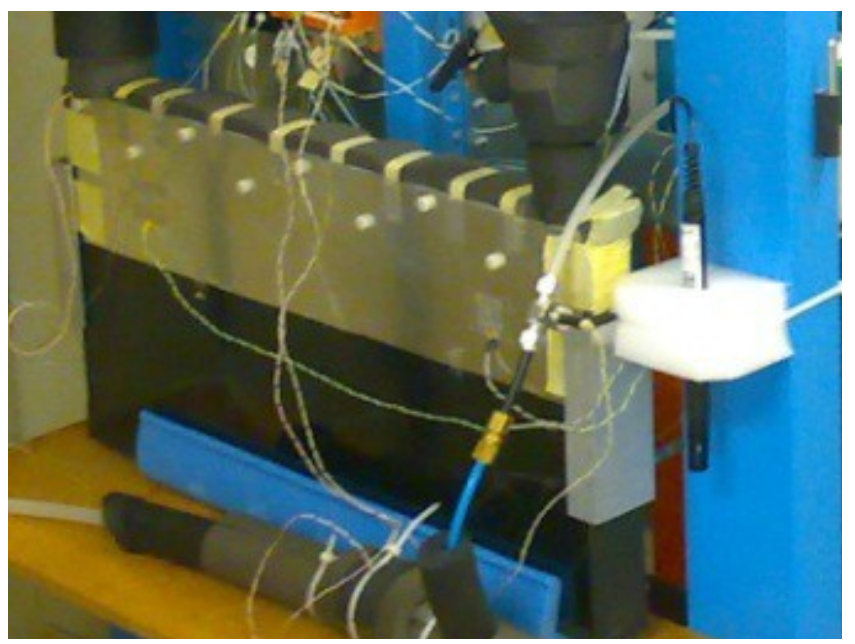




\section{Electronics}

- many of the existing integrated front-end electronics chips for SiPMs optimized for calorimeter readout

- instead of large dynamic range, good linearity, fast sampling and readout are needed

- it was decided to develop an optimized ASIC to read out the SiPMs with $40 \mathrm{MHz}$

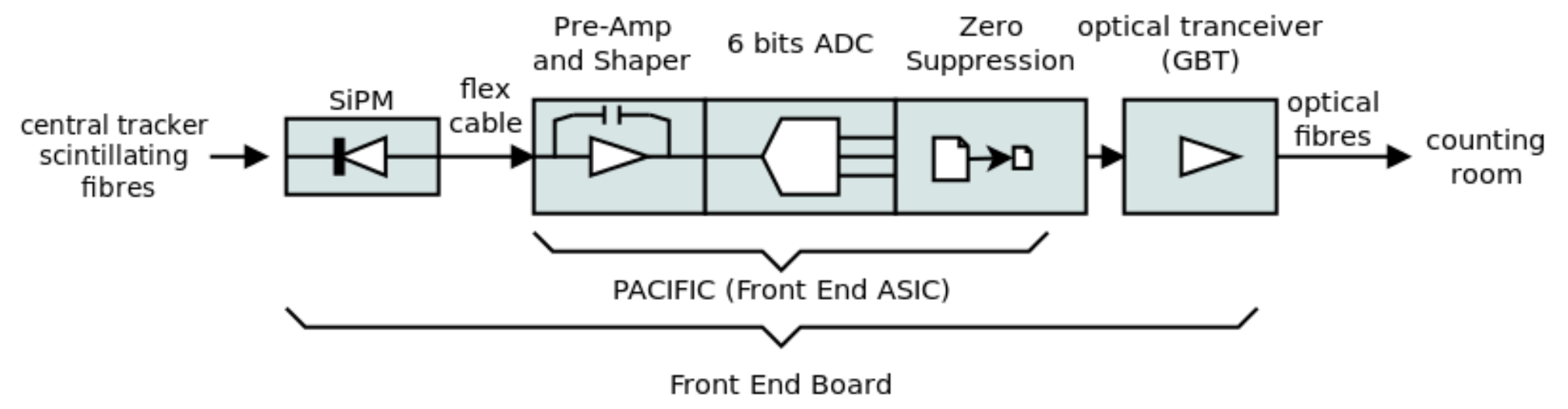




\section{Simulation}

- simulate all steps from light attenuation to tracking

- possible to test the influence of different parameters on tracking performance, e.g.

- detector layouts (distances between layers, mono-layer vs bi-layer)

- dead regions

- stereo angles

- radiation damage

- misalignment

- timing of signal (fibres, SiPMs, electronics) 


\section{Summary}

- radiation environment is an important issue for a scintillating fibre tracker with SiPM readout at LHCb, but damage is understood and can be coped with

- R\&D towards the demonstrator modules is on the home stretch

- after the technology choice the work will continue towards the TDR in March 2014 